\title{
The Feeney Laboratory
}

\section{Department of Immunology, The Scripps Research Institute, La Jolla, CA, USA}

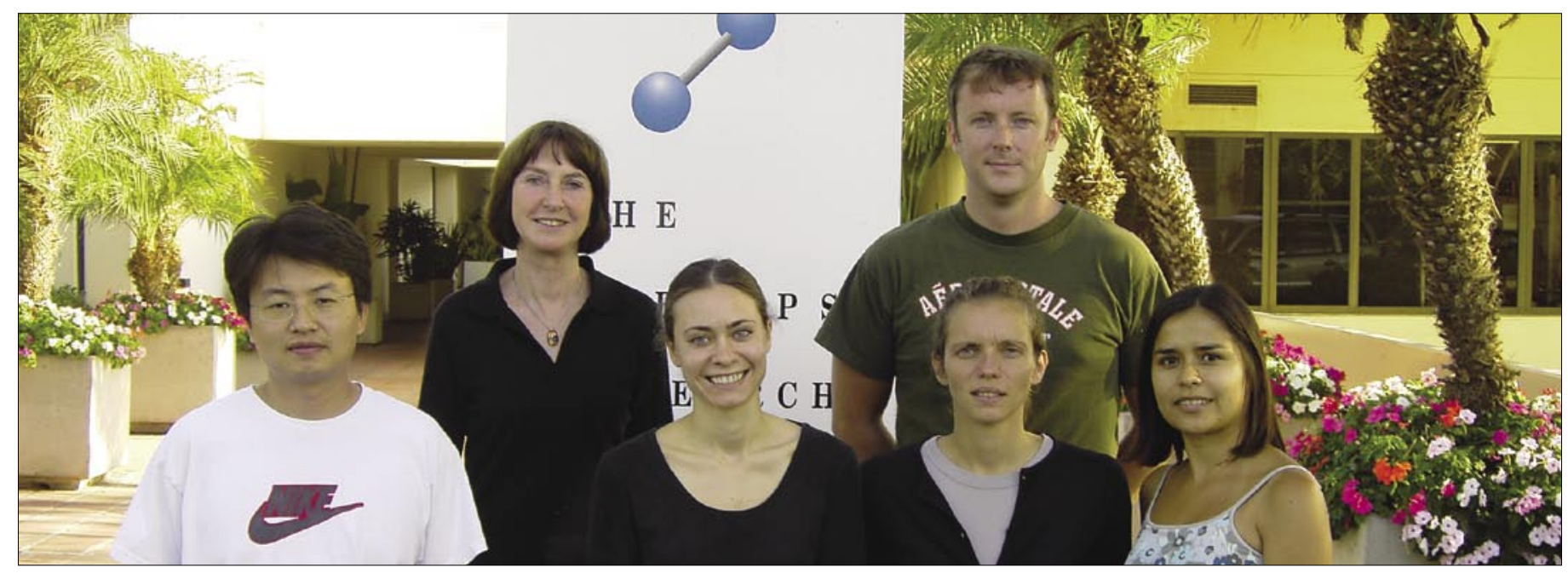

The People: Back row from left: Ann Feeney (Associate Professor) and John Carey (Postdoctoral Research Associate). Front row from left: Cheng-Ran Xu (Postdoctoral Research Associate), Sara Salerno (Research Assistant), Marie Cherrier (Postdoctoral Research Associate), and Celia Espinoza (Senior Postdoctoral Research Associate).

\section{The Research}

Our lab has a long-standing interest in analyzing the factors which control diversity of the primary antibody repertoire. V, D, and J gene segments rearrange at very different frequencies, and we have been analyzing the reasons for this difference. We have shown that the natural variation in the recombination signal sequences (RSS) flanking each gene segment affects recombination frequency, but it does not explain all of the nonrandom rearrangements. Genes with identical RSS can rearrange at very different frequencies, and there is a stronger correlation between rearrangement frequency and the relative position of individual gene segments within the locus. Our recent efforts have centered on the study of the changes in chromatin structure flanking each gene segment. We have shown that the extent of histone H3 and $\mathrm{H} 4$ acetylation of individual $\mathrm{V}$ genes varies and correlates with rearrangement frequency. Furthermore, there is an inverse correlation between rearrangement frequency and the association of $\mathrm{V}$ genes with the repressive H3K9me2 modification. We are currently performing a detailed analysis of the histone methylation and acetylation profiles at many stages during B cell differentiation to elucidate the dynamic pattern of histone posttranslational modifications that control the highly regulated accessibility of receptor gene segments.

\section{The Technique}

Restriction enzyme cleavage of intact nuclei is used to measure chromatin accessibility at specific genomic positions. However, none of the techniques currently used to detect chromatin cleavage have been designed to measure the differential chromatin accessibility of individual members of a multigene family. We have established a chromatin accessibility assay based on ligation-mediated PCR (LM-PCR) and real-time PCR technologies, which allow the sensitive and quantitative measurement of chromatin accessibility of individual members from a highly homologous gene family. In addition, this method requires a small number of cells, which is critical when chromatin accessibility studies are performed on primary cells. We have used this method to evaluate the chromatin status of genomic regions which seem to differentiate frequently from infrequently rearranging immunoglobulin $\mathrm{VH}(\mathrm{IgVH})$ genes in freshly isolated mouse pro-B cells.

Quantifying chromatin accessibility of individual gene family members by combining ligation-mediated PCR with real-time PCR, p. 404.

\section{www.scripps.edu/imm/feeney}

